\title{
What does TGA approval of medicines mean?
}

\author{
John McEwen, Principal Medical Adviser, Therapeutic Goods Administration, Canberra
}

\section{Summary}

The Therapeutic Goods Administration is a Commonwealth Government agency that regulates medical devices and drugs. Prescription medicines and over-the-counter medicines which meet Australian standards of quality, safety and efficacy are included on the Australian Register of Therapeutic Goods. Medicines may be registered or listed. Registered products are thoroughly evaluated and are labelled with an AUST R number. Listed products, such as complementary medicines, do not have to undergo the same assessments and are labelled with an AUST L number. They are not routinely evaluated before marketing, but are subject to a random audit after listing. Some medicines, such as those compounded for individual patients, are not regulated.

Key words: drug industry, drug regulation.

(Aust Prescr 2004;27:156-8)

\section{Introduction}

The Therapeutic Goods Administration (TGA) is a division of the Australian Department of Health and Ageing. Its principal role is as the national regulator of therapeutic goods - a collective term covering medicines, medical devices and some related products. The TGA administers the Therapeutic Goods Act 1989, the objects of which include 'a national system of controls relating to the quality, safety, efficacy and timely availability of therapeutic goods that are used in Australia, whether produced in Australia or elsewhere, or exported from Australia'. These activities are fully funded by fees charged for assessments, annual registrations and inspections.

\section{Australian Register of Therapeutic Goods}

The lawful supply of any therapeutic good in Australia requires that the product is included on the Australian Register of Therapeutic Goods (ARTG). There are two types of entry on the ARTG. Some products are 'registered' and have an AUST R number on their label. Other products are 'listed' and have an AUST L number. The TGA decides if products are suitable for listing or require registration. Manufacturers are not permitted to suggest or imply in their advertisements that inclusion in the ARTG is a recommendation or endorsement by the TGA.

\author{
AUST R products \\ Medicines that are registered include: \\ - almost all prescription medicines \\ - a number of products, such as vaccines, which although not \\ classified in law as needing a prescription warrant detailed \\ evaluation \\ - almost all conventional over-the-counter medicines including, \\ for example, packs of aspirin and paracetamol tablets sold \\ from supermarkets \\ - a very small number of complementary medicines where \\ the TGA has been satisfied that specific claims of efficacy \\ in treatment or prevention of a disease are supported by \\ adequate evidence.
}

The approval of AUST R products is based on satisfactory assessments of their quality, efficacy and safety.

\section{Prescription medicines}

The Australian system for the pre-registration evaluation of new active substances, as well as such things as new routes of administration and the extensions of approved uses ('indications') of already marketed products, has evolved since it was established in 1963. Most prescription medicines in use currently have been evaluated through this system. Nowadays an application for registration of a new active substance must be supported by extensive information about the synthesis of the substance, the method of manufacture of the dose forms, studies of its pharmacology and toxicology in animals and clinical trials in humans demonstrating the efficacy and safety of the product in its proposed use. In addition, certification that manufacture has complied with Good Manufacturing Practice is obligatory.

Registration in Australia does not expire. A product remains registered unless there are grounds for cancellation or the sponsor ceases marketing. A small number of active substances, such as aspirin, were supplied in Australia long before any evaluation process was in place. Their registration is not reviewed unless a safety issue arises or a change in use is proposed.

Many of the prescription medicines used in Australia are versions of the innovator product, usually produced by other manufacturers. These generic products are subject to the same regulation of manufacture and quality standards. However, only evidence that the formulation is bioequivalent to the innovator product is required, rather than a full demonstration of 
efficacy and safety. ${ }^{1}$ Bioequivalence studies usually involve a comparative study of the product in human volunteers, but benchtop testing of dissolution may suffice for some products. Similar testing in human volunteers is required to support the claims of modified-release formulations.

\section{Over-the-counter medicines}

Nowadays, almost all active substances in non-prescription medicines first enter the market as ingredients of prescription medicines. To assess whether or not an active substance is suitable for use in a non-prescription medicine usually requires the substance to have been used for at least two years as a prescription medicine. Not all active substances make the transition from prescription to over-the-counter use. The volume of new information to support efficacy and safety is usually less, because the registration of the over-the-counter product can draw on the accumulated experience as a prescription product. New over-the-counter products are assessed by theTGA for quality, efficacy and safety. The standards for such things as quality and circumstances of manufacture are essentially the same as those of prescription medicines.

\section{AUST L products}

The group of medicines that are listed consists almost entirely of complementary medicines. These include herbal medicines, most vitamin and mineral supplements, other nutritional supplements, traditional medicines such as Ayurvedic medicines and traditional Chinese medicines, and aromatherapy oils. ${ }^{2}$

This category of listed products came into effect in 1991 as a means of regulating products that seemed by their nature to have a low risk of causing adverse effects. Similar requirements for manufacture, including certification of Good Manufacturing Practice, apply as to AUST R products, but they are not evaluated before inclusion in the ARTG. The principal mechanism for ensuring that these products are safe is through the requirements of the Therapeutic Goods Regulations 1990. AUST L medicines must:

- not contain substances that are prohibited imports, come from endangered species or be covered by the national regulations which control access to many substances (Standard for the Uniform Scheduling of Drugs and Poisons)

- conform with lists of permitted ingredients (minerals, vitamins, declared listable substances).

In some instances, there are additional requirements such as dose limits, specified label warnings and limits on plant parts or methods of preparation. Certain herbs are not permitted.

The initial approach to regulation of AUST L products did not require evidence to support manufacturers' claims, provided the products were not for the treatment of serious illnesses.
A concern that multiple and at times improbable claims were being made about products led to the introduction in April 1999 of a requirement that sponsors of AUST L products must hold evidence to substantiate their claims. This evidence may be called for and evaluated by theTGA, should a concern or complaint arise at any time during the life of a product. If the evidence is inadequate, the TGA may cancel the listing for the product. A random sample of approximately $20 \%$ of new listings are assessed in detail for compliance with the listing requirements.

In 2003 an expert committee recommended that sponsors of AUST L medicines should submit summaries of the evidence they hold to support the efficacy of their products, and that the TGA should randomly audit this information. ${ }^{3}$ Where there is evidence to support the efficacy of an AUST L medicine in a serious illness, registration (AUST R status) can be sought.

\section{Exemptions}

Medicines (except for gene therapy) that are dispensed or extemporaneously compounded for a particular person are currently exempt from TGA regulation. Some clinics and pharmacists are using this exemption as a means for supplying very large numbers of patients with medicines made in those pharmacies. On occasions, claims about special characteristics such as 'slow release product' are made. Such products are not assessed or regulated by the TGA. Similar exemptions apply to medicines individually dispensed by traditional Chinese medicine and homeopathic practitioners. ${ }^{4}$

Some other medicines are also exempt from the requirement for inclusion in the ARTG. Perhaps the most important are homeopathic medicines. This exemption from TGA regulation has seen the marketing of such purported homeopathic products as homeopathic somatropin and homeopathic melatonin. IncreasedTGA regulation of homeopathic products has therefore been recommended. ${ }^{3}$ This might be expected to focus on ensuring that such products are formulated with regard to homeopathic principles and practices and are made in compliance with the same manufacturing requirements as conventional medicines.

\section{References}

1. Birkett DJ. Generics - equal or not? Aust Prescr 2003;26:85-7.

2. Cameron $\mathrm{H}$. The regulation of complementary medicines by the Therapeutic Goods Administration. Aust Prescr 1998;21:107-8.

3. Expert committee on complementary medicines in the health system. Report to the Parliamentary Secretary to the Minister for Health and Ageing. Complementary medicines in the Australian health system. Canberra: Commonwealth of Australia; 2003. pp. 85, 88-9.

http://www.tga.gov.au/docs/pdf/cmreport.pdf [cited 2004 Nov 8] 
4. McEwen J, Cumming F. The quality and safety of traditional Chinese medicines [comment]. Aust Prescr 2003;26:130-1.

\section{Further information}

Medicines regulation and the TGA. Canberra:Therapeutic Goods Administration; 2004.

http://www.tga.gov.au/docs/html/medregs.htm [cited 2004 Nov 8]

The Australian Register of Therapeutic Goods

Requests for searches and reports Phone 1800010624

http://www.tga.gov.au/docs/html/artg.htm [cited 2004 Nov 8]

Conflict of interest: none declared

\section{Self-test questions}

The following statements are either true or false (answers on page 165)

5. The Therapeutic Goods Administration routinely evaluates the quality, safety and efficacy of all new products listed on the Australian Register of Therapeutic Goods.

6. Complementary medicines are not regulated by the Therapeutic Goods Administration.

\section{Book review}

Pocket guide to chest X-rays. G. Briggs.

Sydney: McGraw-Hill Australia; 2004.

\section{2 pages. Price $\$ 32.95$ including GST*}

\section{Lindy Viviers, Senior Resident Medical Officer, Division of Medicine, John Hunter Hospital, Newcastle, NSW}

For a quick revision of the basics of chest X-ray interpretation, as well as some more advanced tips, 'Pocket guide to chest X-rays' by Greg Briggs is a useful addition to the clinician's library. The guide begins with a summary of the major radiological modalities and their indications in practice. It explains the techniques of chest radiography in easily understood prose with accompanying diagrams. This is followed by a section on radiological anatomy and a thorough description of normal chest X-rays and variants. It thus offers the reader the chance to consolidate their knowledge of 'the norm' with which to compare abnormal X-rays presented in the remainder of its pages.

The book endeavours to be a practical guide to be used as an adjunct to a physician's practice. It outlines a systematic checklist with which to approach all chest $\mathrm{X}$-rays and this is probably one of its foremost strengths. The bulk of the book is a collection of actual chest X-rays that showcase the common pathologies which clinicians encounter. For a large majority of students and trainees a picture can speak a thousand words and this book offers approximately $50 \mathrm{X}$-rays of conditions seen in everyday practice. The descriptions of these are straightforward and easy to follow. A noteworthy inclusion is a list of common pitfalls in interpreting chest X-rays, at least one or two of which the honest clinician will recognise.

The appendices are also worth mentioning. One is devoted to various signs in thoracic radiology, which, while being very detailed in its descriptions, would probably be more useful if accompanied by the actual radiological pictures. Nonetheless, a number of these signs are referred to in the body of the book and it is a matter of looking them up. The second appendix is simply a quick reference list of causes and differential diagnoses commonly encountered by doctors. The last is an alphabetical list of syndromes particularly relevant to chest radiology, some of which are more recognisable than others, and would probably spur a number of us to revisit our textbooks.

This guide's main use is as a reference in the context of patient care, however the medical student would find it invaluable as a learning tool as well. On the whole this is an easy, informative read that encapsulates a rather enormous area of medicine into a concise, manageable whole.

* Australian Prescriber readers are offered 15\% discount by McGraw-Hill Australia (phone (02) 99001806 or email cservice_sydney@mcgraw-hill.com and quote code BCX15).

\section{Book review policy}

Publishers sometimes ask Australian Prescriber to review their books, CDs and web sites. The Editorial Executive Committee considers these requests according to Australian Prescriber's book review policy:

1. Books are reviewed if they are likely to be of interest to readers of Australian Prescriber. Not all books are reviewed.

2. Reviewers are selected according to their areas of expertise.

3. Reviewers must declare any conflicts of interest.

4. Reviewers are not paid, but may keep the review copy.

5. Book reviews may be edited.

6. Not all reviews are published.

7. No payments or commissions are accepted for these reviews from publishers. 